\title{
Scattering Study of Fermions due to Double Dirac Delta Potential in Quaternionic Relativistic Quantum Mechanics
}

\author{
Hassan Hassanabadi, ${ }^{1}$ Hadi Sobhani ${ }^{1},{ }^{1}$ and Won Sang Chung ${ }^{2}$ \\ ${ }^{1}$ Faculty of Physics, Shahrood University of Technology, P.O. Box 3619995161-316, Shahrood, Iran \\ ${ }^{2}$ Department of Physics and Research Institute of Natural Science, College of Natural Science, Gyeongsang National University, \\ Jinju 52828, Republic of Korea
}

Correspondence should be addressed to Hadi Sobhani; hadisobhani8637@gmail.com

Received 21 September 2017; Revised 15 November 2017; Accepted 23 November 2017; Published 3 January 2018

Academic Editor: Edward Sarkisyan-Grinbaum

Copyright (C) 2018 Hassan Hassanabadi et al. This is an open access article distributed under the Creative Commons Attribution License, which permits unrestricted use, distribution, and reproduction in any medium, provided the original work is properly cited. The publication of this article was funded by SCOAP S $^{3}$

We have studied the scattering problem of relativistic fermions from a quaternionic double Dirac delta potential. We have used Dirac equation in the presence of the scalar and vector potentials in the quaternionic formalism of relativistic quantum mechanics to study the problem. The wave functions of different regions have been derived. Then, using the reflection coefficient, transmission coefficient, and the continuity equation, the scattering problem has been investigated in detail. It has been shown that we have faced some fluctuations in the reflection and transmission coefficients.

\section{Introduction}

Among a lot of mathematical options in physics, quaternions are a famous option. Quaternion was initiated by Hamilton for the first time [1-3]. Quaternions in general can be represented by

$$
\phi=\phi_{0}+\phi_{1} e_{1}+\phi_{2} e_{2}+\phi_{3} e_{3}
$$

where $\phi_{l}(l=0,1,2,3)$ are real coefficients. There are three imaginary units in quaternions which have the property

$$
e_{a} e_{b}=-\delta_{a b}+\varepsilon_{a b c} e_{c}, \quad(a, b, c=1,2,3) .
$$

If we set $i, j, k$ as the imaginary units, we conclude from (2) that

$$
\begin{gathered}
i j=-j i=k, \\
j k=-k j=i, \\
k i=-i k=j .
\end{gathered}
$$

Equation (3) exhibits that in general multiplication of two quaternions has noncommutative nature; it means $q p \neq p q$.
The idea for making use of quaternions in quantum mechanics has a long shining story. Physicists and mathematician have tried to found quantum mechanics on the quaternions. Finkelstein et al. discussed a new kind of quantum mechanics by using quaternions [4] or some works which are about using real and complexified quaternions as underlying mathematical structure by adopting a complex geometry to have ability to present a compatible face of quantum mechanics $[5,6]$; tunneling phenomena for the relativistic fermions have been studied in the presence of quaternionic step potential $[7,8]$ and there are a lot of valuable researches in this topic which are mentioned in [9-17]. All of them tried to show that we can have a quantum mechanics based on the quaternions. In the rest of the paper, we intend to present quaternionic form of Dirac equation. We want to extend our recent papers [18-21] to relativistic form as well as having a study of scattering by double Dirac delta potential. In our recent paper [20], we derived the Dirac equation in quaternionic quantum mechanics, in general. Then scattering of relativistic fermions due to a single quaternionic Dirac delta function has been studied. It was seen that the reflection and transmission coefficients were treated smoothly and without any fluctuation. But it will be shown that scattering due to the quaternionic double Dirac delta function results 
in some fluctuations in the reflection and transmission coefficients. We organized this article as presenting quaternionic Dirac equation in Section 2 and introducing the quaternionic double Dirac delta potential and the discussion about it and its effects in Section 3, and after bringing some information about probability current and conservation law in Section 4, the conclusions appeared at the end.

\section{Dirac Equation in Quaternionic Relativistic Quantum Mechanics}

As in the ordinary relativistic quantum mechanics, to investigate relativistic fermions, we should make use of Dirac equation. Quaternionic version of Dirac equation in the presence of vector and scalar potential can be written with the help of [8] as

$$
\begin{aligned}
& \frac{\partial \Psi(\mathbf{r}, t)}{\partial t}=-\left[\boldsymbol{\alpha} \cdot \nabla+\boldsymbol{\beta}\left(i\left(m+S_{a}(\mathbf{r})\right)+j S_{b}(\mathbf{r})\right)\right. \\
& \left.\quad+i V_{a}(\mathbf{r})+j V_{b}(\mathbf{r})\right] \Psi(\mathbf{r}, t)
\end{aligned}
$$

where in $(4) \hbar=c=1$ and

$$
\begin{aligned}
& \alpha=\left(\begin{array}{ll}
0 & \sigma \\
\sigma & 0
\end{array}\right) \\
& \beta=\left(\begin{array}{cc}
1 & 0 \\
0 & -1
\end{array}\right),
\end{aligned}
$$

where Pauli matrices are $\boldsymbol{\sigma}$. As is obviously clear, the potentials have two parts. Real functions of them are shown by subscribing $a\left(S_{a}(\mathbf{r}), V_{a}(\mathbf{r}) \in \mathbb{R}\right)$ and for complex functions, they have subscribed $b\left(S_{b}(\mathbf{r}), V_{b}(\mathbf{r}) \in \mathbb{C}\right)$. By setting $S_{b}(\mathbf{r}), V_{b}(\mathbf{r}) \rightarrow$ 0 , we can get the well-known form of this equation as $[22,23]$

$$
i \frac{\partial \Psi(\mathbf{r}, t)}{\partial t}=\left(\boldsymbol{\alpha} \cdot \mathbf{P}+\boldsymbol{\beta}\left(m+S_{a}(\mathbf{r})\right)+V_{a}(\mathbf{r})\right) \Psi(\mathbf{r}, t) .
$$

Since we are interested in the time-independent interactions, it is better to consider wave function as

$$
\Psi(\mathbf{r}, t)=\Phi(\mathbf{r}) e^{-i E t} .
$$

Inserting (7) into (4), the time-independent form of quaternionic Dirac equation derives as

$$
\begin{aligned}
& \Phi(\mathbf{r}) i E=\left(\boldsymbol{\alpha} \cdot \nabla+\boldsymbol{\beta}\left(i\left(m+S_{a}(\mathbf{r})\right)+j S_{b}(\mathbf{r})\right)+i V_{a}(\mathbf{r})\right. \\
& \left.\quad+j V_{b}(\mathbf{r})\right) \Phi(\mathbf{r}) .
\end{aligned}
$$

Note the coordinate part of wave function is a quaternionic function and has two components. Actually it is usually written like

$$
\Phi(\mathbf{r})=\left(\begin{array}{l}
\Phi^{+}(\mathbf{r}) \\
\Phi^{-}(\mathbf{r})
\end{array}\right)
$$

where $\Phi^{ \pm}(\mathbf{r})$ are quaternionic functions. This form of representing is called spinor form of wave function. If we set $i S_{a}(\mathbf{r})+j S_{b}(\mathbf{r})=i V_{a}(\mathbf{r})+j V_{b}(\mathbf{r})$, by doing some algebraic calculations, we arrive at a coupled system of equations for the components

$$
\begin{aligned}
\Phi^{+}(x) i E= & \sigma_{x} \frac{d \Phi^{-}(x)}{d x} \\
& +\left(i m+2\left(i S_{a}(x)+j S_{b}(x)\right)\right) \Phi^{+}(x), \\
\Phi^{-}(x) i E= & \sigma_{x} \frac{d \Phi^{+}(x)}{d x}-i m \Phi^{-}(x) .
\end{aligned}
$$

Quaternionic wave function components are in the form of $\Phi^{ \pm}(x)=\phi_{a}^{ \pm}(x)+j \phi_{b}^{ \pm}(x)$, where $\phi_{a}^{ \pm}(x)$ and $\phi_{b}^{ \pm}(x)$ are the complex functions. Considering such a form of the wave function components in (11) yields

$$
\begin{aligned}
& \phi_{a}^{-}(x)=\frac{\sigma_{x}}{i(E+m)} \frac{d \phi_{a}^{+}(x)}{d x}, \\
& \phi_{b}^{-}(x)=\frac{\sigma_{x}}{i(E-m)} \frac{d \phi_{b}^{+}(x)}{d x} .
\end{aligned}
$$

Substitution of (12) into (10) provides a system of coupled differential equations

$$
\begin{aligned}
& \frac{d^{2} \phi_{a}^{+}(x)}{d x^{2}}+\left(p^{2}-2(E+m) S_{a}(x)\right) \phi_{a}^{+}(x) \\
& \quad-2 i(E+m) S_{b}^{*}(x) \phi_{b}^{+}(x)=0, \\
& \frac{d^{2} \phi_{b}^{+}(x)}{d x^{2}}+\left(-p^{2}-2(E-m) S_{a}(x)\right) \phi_{b}^{+}(x) \\
& \quad+2 i(E-m) S_{b}(x) \phi_{a}^{+}(x)=0,
\end{aligned}
$$

where $p^{2}=E^{2}-m^{2}$ and $*$ means the complex conjugation. Now, we are in a position to investigate scattering states due to quaternionic Dirac delta potential.

\section{Quaternionic Double Dirac Delta and Scattering}

Here, we introduce a quaternionic form of double Dirac delta interaction [21] as

$$
\begin{aligned}
& S_{a}(x)=V_{a}\left(\delta\left(x-a_{0}\right)+\delta\left(x+a_{0}\right)\right) \\
& S_{b}(x)=i V_{b}\left(\delta\left(x-a_{0}\right)+\delta\left(x+a_{0}\right)\right) .
\end{aligned}
$$

We assume that $V_{a}$ and $V_{b}$ are real constants. The wellknown property of Dirac delta interactions is producing discontinuity condition for the derivative of wave function. These conditions can be derived by integrating (13) around $x=a_{0}$ and $x=-a_{0}$. So discontinuity condition at $x=a$ is

$$
\begin{aligned}
& \left.\frac{d \phi_{a}^{+}}{d x}\right|_{x=a_{0}^{+}}-\left.\frac{d \phi_{a}^{+}}{d x}\right|_{x=a_{0}^{-}} \\
& =2(E+m)\left(V_{a} \phi_{a}^{+}\left(a_{0}\right)+V_{b} \phi_{b}^{+}\left(a_{0}\right)\right), \\
& \left.\frac{d \phi_{b}^{+}}{d x}\right|_{x=a_{0}^{+}}-\left.\frac{d \phi_{b}^{+}}{d x}\right|_{x=a_{0}^{-}} \\
& =2(E-m)\left(V_{a} \phi_{a}^{+}\left(a_{0}\right)+V_{b} \phi_{b}^{+}\left(a_{0}\right)\right) .
\end{aligned}
$$


Also for $x=-a$, we have

$$
\begin{aligned}
& \left.\frac{d \phi_{a}^{+}}{d x}\right|_{x=-a_{0}^{+}}-\left.\frac{d \phi_{a}^{+}}{d x}\right|_{x=-a_{0}^{-}} \\
& =2(E+m)\left(V_{a} \phi_{a}^{+}\left(-a_{0}\right)+V_{b} \phi_{b}^{+}\left(-a_{0}\right)\right), \\
& \left.\frac{d \phi_{b}^{+}}{d x}\right|_{x=-a_{0}^{+}}-\left.\frac{d \phi_{b}^{+}}{d x}\right|_{x=-a_{0}^{-}} \\
& =2(E-m)\left(V_{a} \phi_{a}^{+}\left(-a_{0}\right)+V_{b} \phi_{b}^{+}\left(-a_{0}\right)\right) .
\end{aligned}
$$

By the way, we should make three parts in our problem

$$
\begin{aligned}
\text { Region I } & x<-a_{0}, \\
\text { Region II } & -a_{0}<x<a_{0}, \\
\text { Region III } & x>a_{0} .
\end{aligned}
$$

For the free particles, we have

$$
\begin{aligned}
& \phi_{a}^{+}(x)=c_{1} e^{i p x}+c_{2} e^{-i p x} \\
& \phi_{b}^{+}(x)=c_{3} e^{p x}+c_{4} e^{-p x}
\end{aligned}
$$

where the coefficients are complex constants spinors in general. Therefore, according to our assumption about the particles, the physical wave functions can be written as

$$
\begin{aligned}
\phi_{\mathrm{I}}^{+}(x) & =e^{i p x}+r e^{-i p x}+j e^{p x}, \\
\phi_{\mathrm{II}}^{+}(x) & =c_{1} e^{i p x}+c_{2} e^{-i p x}+j\left(c_{3} e^{p x}+c_{4} e^{-p x}\right), \\
\phi_{\mathrm{III}}^{+} & =t e^{i p x}+\tilde{j t} e^{-p x} .
\end{aligned}
$$

It can be easily seen that if we remove the coefficient and the imaginary unit $j$, we obtain the wave function of the free relativistic fermions in the ordinary quantum mechanics. In order to have explicit expression of the coefficients in (19), we should match the wave functions at $x=a_{0}$ and $x=-a_{0}$ which yields

$$
\begin{aligned}
& x=a_{0}\left\{\begin{array}{l}
c_{1} e^{i a_{0} p}+c_{2} e^{-i a_{0} p}=t e^{i a_{0} p}, \\
c_{3} e^{a_{0} p}+c_{4} e^{-a_{0} p}=\tilde{t} e^{-a_{0} p},
\end{array}\right. \\
& x=-a_{0}\left\{\begin{array}{l}
r e^{i a_{0} p}+e^{-i a_{0} p}=c_{1} e^{-i a_{0} p}+c_{2} e^{i a_{0} p}, \\
r e^{i a_{0} p}+e^{-i a_{0} p}=c_{1} e^{-i a_{0} p}+c_{2} e^{i a_{0} p},
\end{array}\right.
\end{aligned}
$$

and four equations also are derived by applying the continuity conditions

$$
\begin{aligned}
& x=a_{0}\left\{\begin{array}{l}
-i c_{1} p e^{i a_{0} p}+i c_{2} p e^{-i a_{0} p}+i p t e^{i a_{0} p}=2(E+m)\left(V_{b} \tilde{t} e^{-a_{0} p}+t V_{a} e^{i a_{0} p}\right) \\
p \tilde{t}\left(-e^{-a_{0} p}\right)-c_{3} p e^{a_{0} p}+c_{4} p e^{-a_{0} p}=2(E-m)\left(V_{b} \tilde{t} e^{-a_{0} p}+t V_{a} e^{i a_{0} p}\right)
\end{array}\right. \\
& x=-a_{0}\left\{\begin{array}{l}
i c_{1} p e^{-i a_{0} p}-i c_{2} p e^{i a_{0} p}+i p r e^{i a_{0} p}-i p e^{-i a_{0} p}=2(E+m)\left(V_{b} \widetilde{r} e^{-a_{0} p}+V_{a}\left(r e^{i a_{0} p}+e^{-i a_{0} p}\right)\right) \\
p \widetilde{r}\left(-e^{-a_{0} p}\right)+c_{3} p e^{-a_{0} p}-c_{4} p e^{a_{0} p}=2(E-m)\left(V_{b} \tilde{r} e^{-a_{0} p}+V_{a}\left(r e^{i a_{0} p}+e^{-i a_{0} p}\right)\right) .
\end{array}\right.
\end{aligned}
$$

So we have eight equations and undetermined coefficients. By solving these equations, explicit form of each coefficient can be determined.

\section{Probability Current and Conservation Law}

In the quaternionic quantum mechanics, similar to the complex version, we have the continuity equation as [20]

$$
\frac{\partial \rho}{\partial t}+\nabla \cdot \mathbf{J}=0
$$

where

$$
\begin{aligned}
& \rho=\bar{\Psi} \Psi, \\
& \mathbf{J}=\Psi^{\dagger} \boldsymbol{\alpha} \Psi .
\end{aligned}
$$

To check the conservation law of probability, we need to calculate the currents of each region. To derive the currents of each region, we need the spinor form of the wave function of each region. This form of the wave functions can be obtained using (12) as

$$
\begin{aligned}
& \Phi_{\mathrm{I}}(x)=\left(\begin{array}{c}
e^{i p x}+r e^{-i p x}+j \widetilde{r} e^{p x} \\
\sigma_{x} p\left(\frac{e^{i p x}-r e^{-i p x}}{E+m}+j \frac{\widetilde{r} e^{p x}}{i(E-m)}\right)
\end{array}\right), \\
& \Phi_{\mathrm{II}}(x)=\left(\begin{array}{c}
t e^{i p x}+j \tilde{t} e^{-p x} \\
\sigma_{x} p\left(\frac{t e^{i p x}}{E+m}-j \frac{\tilde{t} e^{-p x}}{i(E-m)}\right)
\end{array}\right) .
\end{aligned}
$$

As is indicated in the Appendix, it is straightforward to prove that, by using the definition $J_{x}=\Psi^{\dagger} \alpha_{x} \Psi$, we derive the constraint

$$
|r|^{2}+|t|^{2}=1
$$

So form of conservation law of probability is (25). This equation has been plotted in Figure 1 considering $m=a_{0}=V_{a}=$ $V_{b}=1$ and $E \in[1,4]$. 

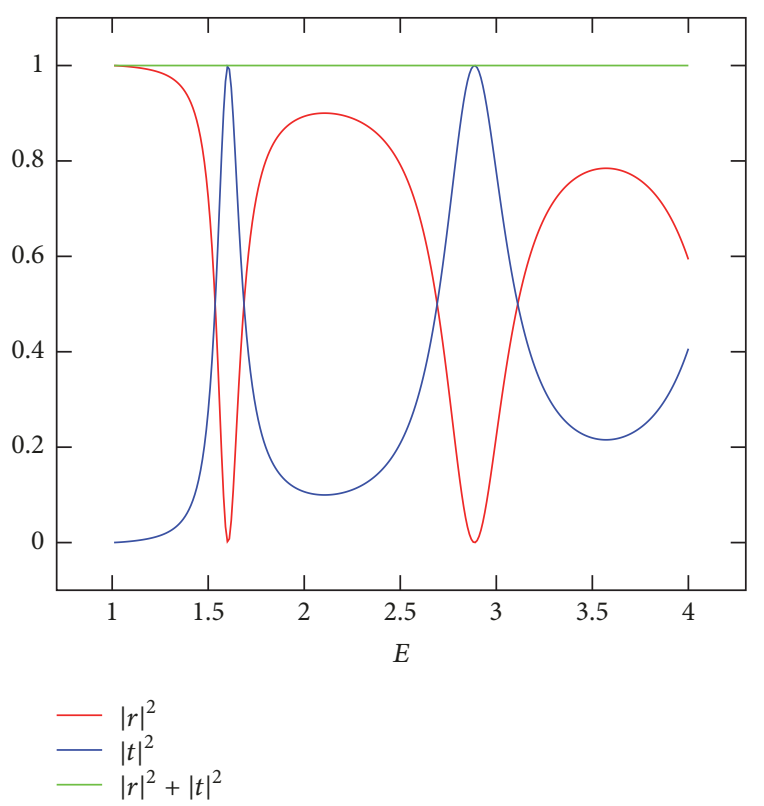

FIGURE 1: Treatments of the coefficients in terms of energy. For this plot, we have set $m=a_{0}=V_{a}=V_{b}=1$ and energy $\epsilon[1,4]$.

As is shown in Figure 1, (25) is valid. It is constructive if we check effects of the potential coefficients and the distance of the Dirac delta functions on the reflection and transmission coefficients. In Figures 2 and 3, the effects of potential coefficients on the reflection and transmission coefficients were shown. It is seen that in Figure 1 by increasing $V_{a}$, the reflection and transmission coefficient appear sharper but in Figure 2 we face a different case. When $V_{b}$ grows up, the reflection and transmission coefficients arise smoother than those in Figure 2.

In Figure 4, by considering fix values for the potential coefficients and the energy, we change the distance between the double Dirac delta functions. It can be seen that by increasing the distance, the number of fluctuations in the reflection and transmissions increases.

\section{Conclusions}

In this paper, we presented quaternionic version of Dirac equation in the presence of vector and scalar potentials. To ensure the correctness of this type of equation, we checked it in special case where we do not have quaternionic potential, and the result was what we expected. After introducing the scattering potential, we investigated effects of it. It causes the discontinuity conditions for the derivative of the wave functions. The probability current for each region of our considered problem was determined. At the end conservation law of probability was derived. How different parameters of the scattering potential can affect the reflection and transmission coefficients was shown; for instance, increasing the first part of the potential causes to have sharper reflection and transmission coefficients but the second part of the potential was treated vice versa. It means that by decreasing the second part of the potential we have the sharp reflection and transmission coefficients. For the last case, effect of the distance between the double Dirac delta functions was shown. It was seen that the number of fluctuations of the reflection and transmission increases by enlarging the distance of the double Dirac delta functions.

\section{Appendix}

In this section, details of derivation of (25) are mentioned. In order to brief the calculation, we will indicate them in a compact form.

At the first step, we are going to derive the current of probability of region I. Using the definition of the probability current, we have [20]

$$
\begin{aligned}
J_{\mathrm{I}} & =\Psi^{\dagger} \alpha_{x} \Psi \\
& =\left(e^{-i p x}+r^{*} e^{i p x}-e^{p x} \widetilde{r}^{*} j, \sigma_{x} p\left(\frac{e^{-i p x}-r^{*} e^{i p x}}{E+m}+\frac{\widetilde{r}^{*} e^{p x}}{i(E-m)} j\right)\right)\left(\begin{array}{cc}
0 & \sigma_{x} \\
\sigma_{x} & 0
\end{array}\right)\left(\begin{array}{c}
e^{i p x}+r e^{-i p x}+j \widetilde{r} e^{p x} \\
\sigma_{x} p\left(\frac{e^{i p x}-r e^{-i p x}}{E+m}+j \frac{\tilde{r} e^{p x}}{i(E-m)}\right.
\end{array}\right) .
\end{aligned}
$$

This point should be noted as the spinor of wave function is a quaternion and since the coefficients are complex constants, their order and the imaginary unit $j$ are important. Hence in daggered form of the spinor, we face a reversed order in (A.1). Proceeding more in the matrix multiplication, we have

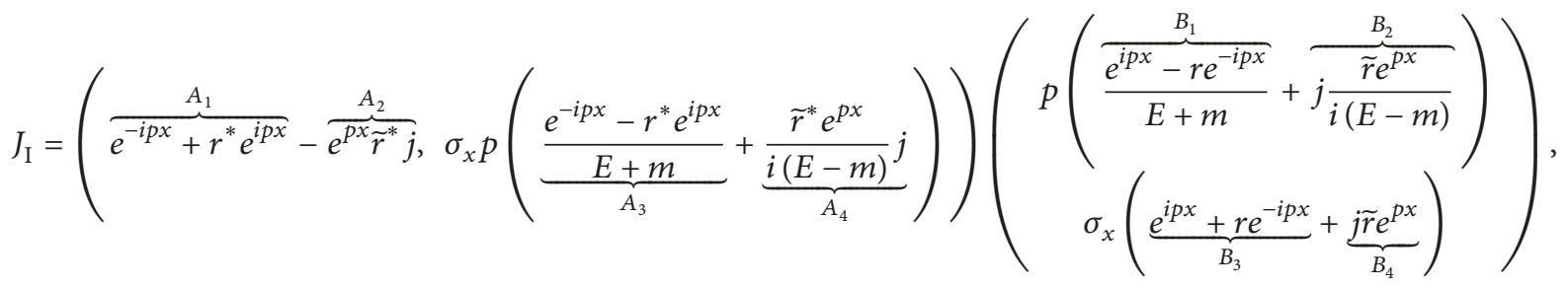

$$
\begin{aligned}
& J_{\mathrm{I}}=A_{1} B_{1}+A_{1} B_{2}+A_{2} B_{1}+A_{2} B_{2}+A_{3} B_{3}+A_{3} B_{4}+A_{4} B_{3}+A_{4} B_{4} \text {. }
\end{aligned}
$$



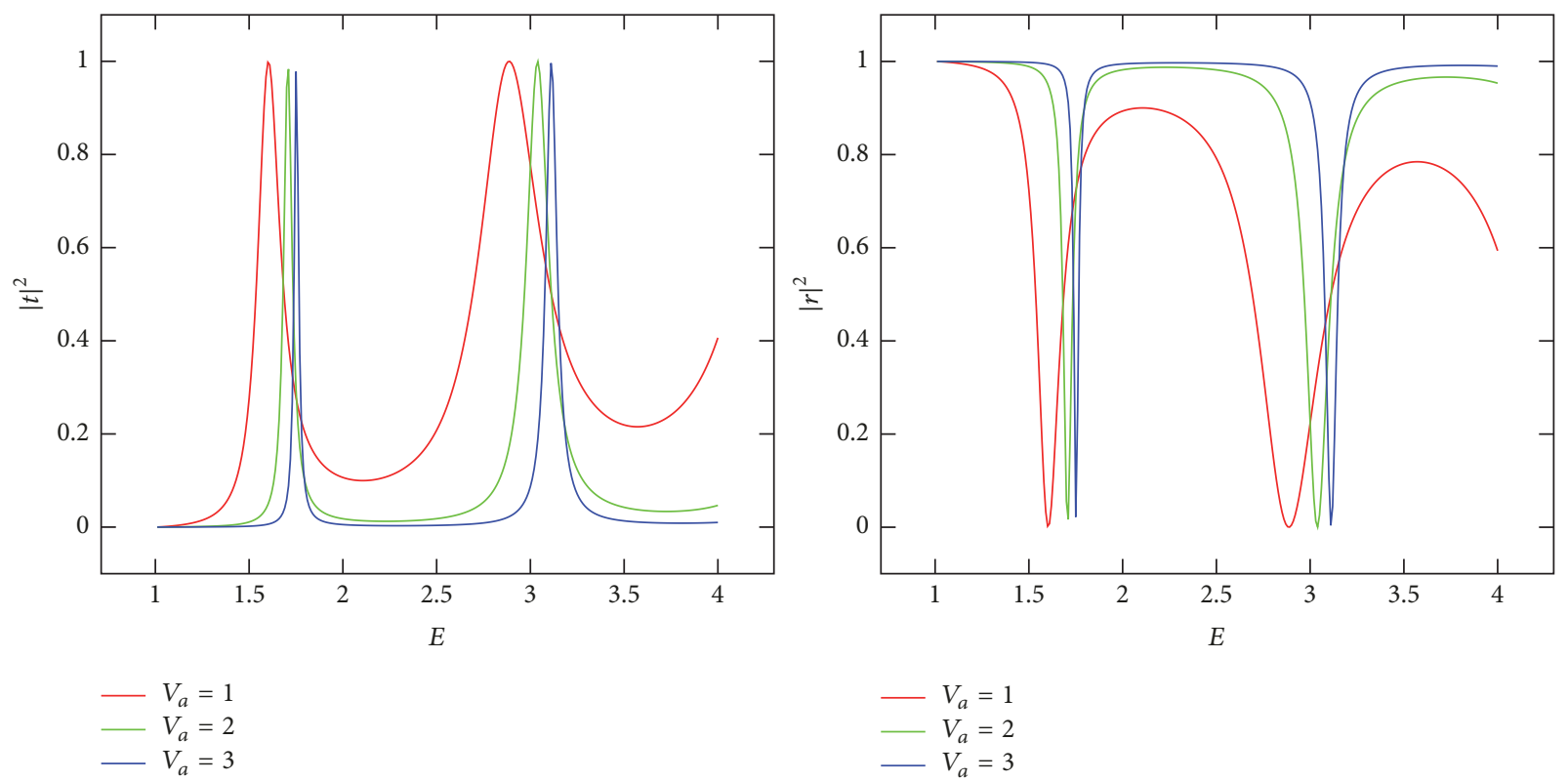

Figure 2: Effects of different values of $V_{a}$ on the coefficients.
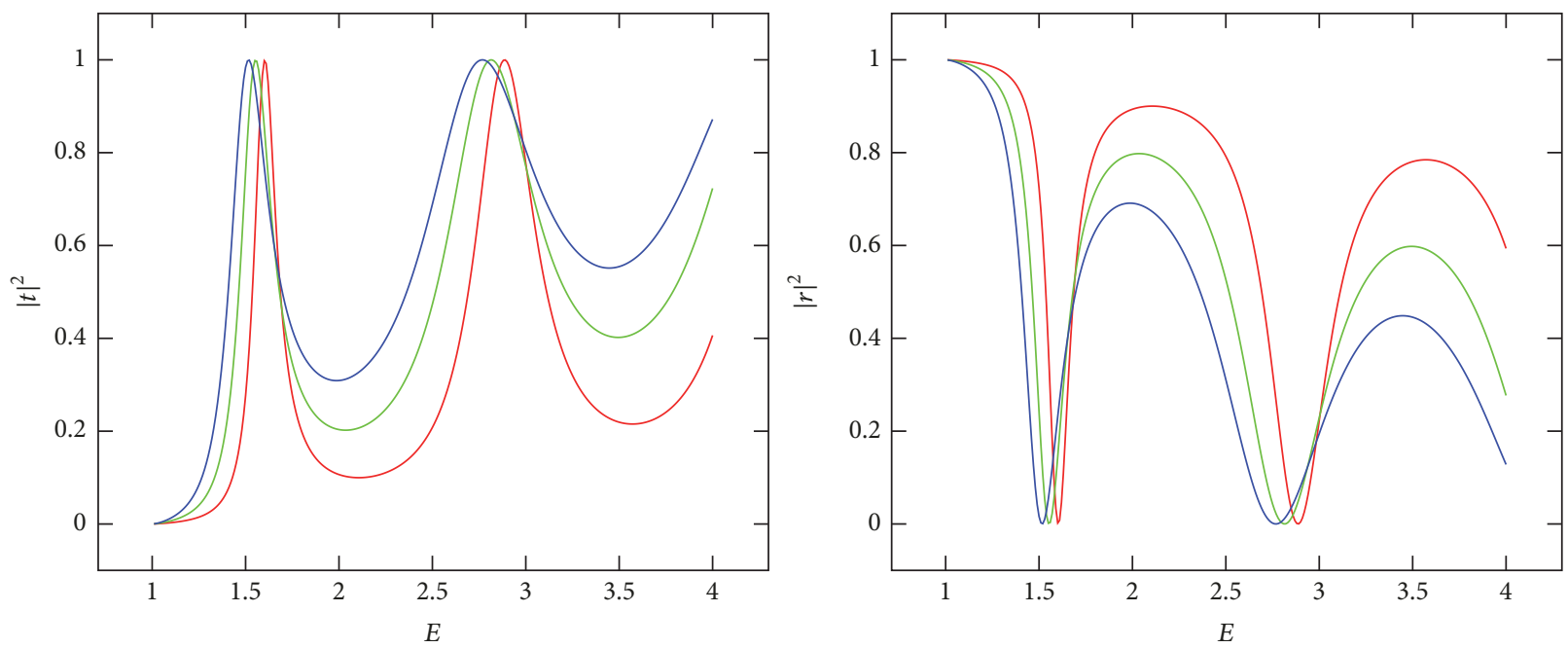

$$
\begin{aligned}
-V_{b} & =1 \\
-V_{b} & =2 \\
-V_{b} & =3
\end{aligned}
$$

$-V_{b}=1$

$-V_{b}=2$

$-V_{b}=3$

FIgURE 3: Effects of different values of $V_{b}$ on the coefficients.

To avoid complicity of multiplication of (A.2), we have

$$
\begin{aligned}
& A_{1} B_{1}=\frac{p}{E+m}\left(1-r e^{-2 i p x}+r^{*} e^{2 i p x}-|r|^{2}\right), \\
& A_{1} B_{2}=j \frac{p}{i(E-m)}\left(\widetilde{r} e^{p x(1+i)}+r \tilde{r} e^{p x(1-i)}\right), \\
& A_{2} B_{1}=j \frac{p}{(E+m)}\left(-\widetilde{r} e^{p x(1+i)}+r \tilde{r} e^{p x(1-i)}\right), \\
& A_{2} B_{2}=\frac{p}{i(E-m)}\left(|\widetilde{r}|^{2} e^{2 p x}\right),
\end{aligned}
$$

$$
\begin{aligned}
& A_{3} B_{3}=\frac{p}{E+m}\left(1+r e^{-2 i p x}-r^{*} e^{2 i p x}-|r|^{2}\right), \\
& A_{3} B_{4}=j \frac{p}{(E+m)}\left(\tilde{r} e^{p x(1+i)}-r \tilde{r} e^{p x(1-i)}\right), \\
& A_{4} B_{3}=-j \frac{p}{i(E-m)}\left(\tilde{r} e^{p x(1+i)}+r \tilde{r} e^{p x(1-i)}\right), \\
& A_{4} B_{4}=-\frac{p}{i(E-m)}\left(|\widetilde{r}|^{2} e^{2 p x}\right) .
\end{aligned}
$$



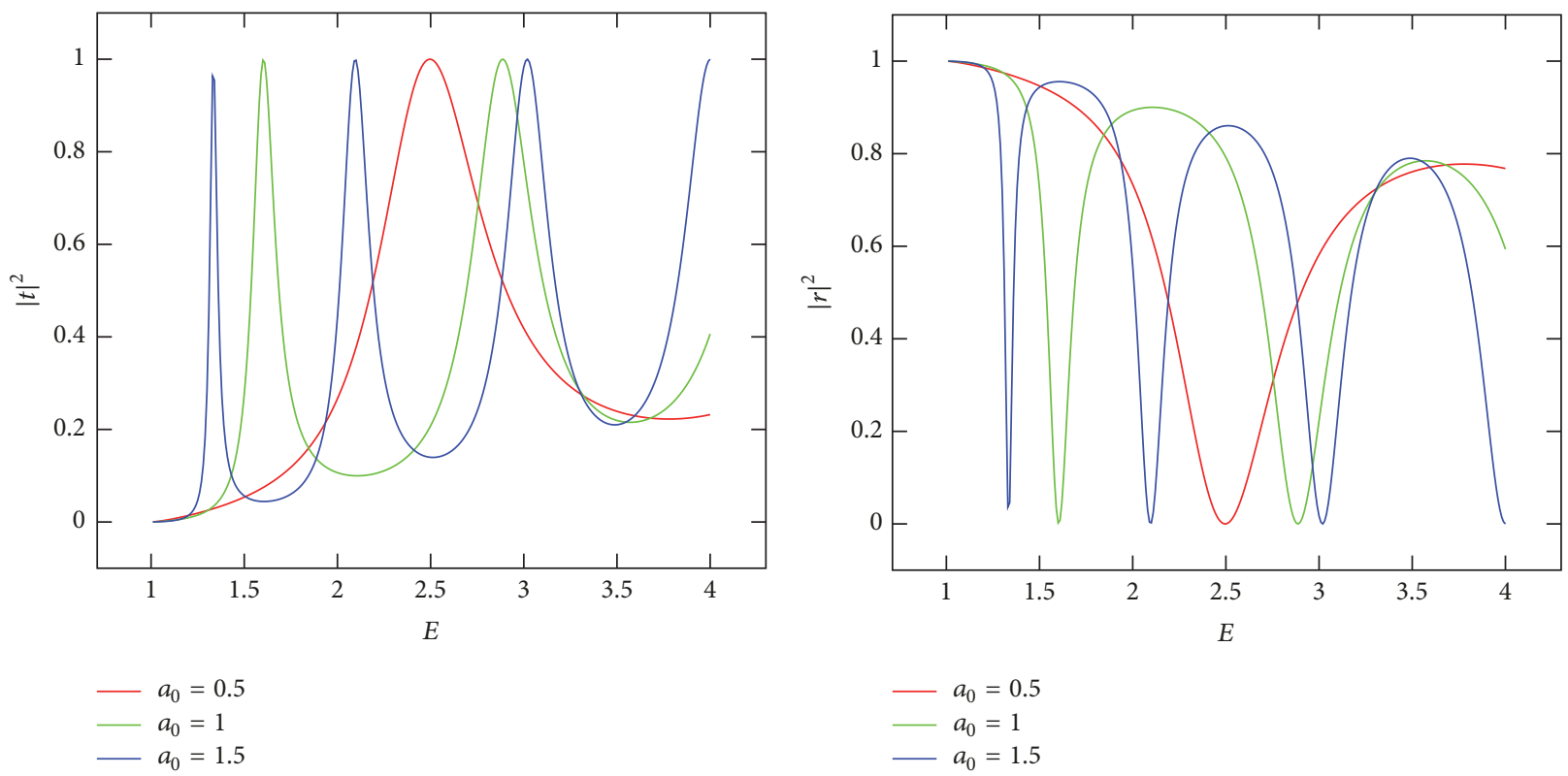

FIGURE 4: Effects of different values of $a_{0}$ on the coefficients.

With the help of (A.3) and (A.4), we can find the probability current of region I as

$$
J_{\mathrm{I}}=\frac{2 p}{E+m}\left(1-|r|^{2}\right)
$$

In the same manner, we can find the probability current of region II as

$$
J_{\mathrm{II}}=\frac{2 p}{E+m}|t|^{2} .
$$

Since there is no sink or source for the particles, we have

$$
\begin{aligned}
J_{\mathrm{I}} & =J_{\mathrm{II}} \Longrightarrow \\
|r|^{2}+|t|^{2} & =1 .
\end{aligned}
$$

\section{Conflicts of Interest}

The authors declare that they have no conflicts of interest.

\section{References}

[1] W. R. Hamilton, Elements of Quaternions, New York, NY, USA, 1969.

[2] W. R. Hamilton, The mathematical papers of Sir William Rowan Hamilton, Cambridge University Press, Cambridge, UK, 1967.

[3] B. A. Rosenfeld, A history of Non-Euclidean Geometry, vol. 12, Springer, New York, NY, USA, 1988.

[4] D. Finkelstein, J. M. Jauch, and D. Speiser, "Quaternionic representations of compact groups," Journal of Mathematical Physics, vol. 4, pp. 136-140, 1963.

[5] J. Rembielinski, "Tensor product of the octonionic Hilbert spaces and colour confinement," Journal of Physics A: Mathematical and General, vol. 11, p. 2323, 1978.
[6] L. P. Horwitz and L. C. Biedenharn, "Quaternion quantum mechanics: second quantization and gauge fields," Annals of Physics, vol. 157, no. 2, pp. 432-488, 1984.

[7] S. De Leo and S. Giardino, "Dirac solutions for quaternionic potentials," Journal of Mathematical Physics, vol. 55, no. 2, 022301, 10 pages, 2014.

[8] S. De Leo, G. Ducati, and S. Giardino, "Quaternionic dirac scattering," Journal of Physical Mathematics, vol. 6, article 1000130, 2015.

[9] S. De Leo and G. Ducati, "Quaternionic differential operators," Journal of Mathematical Physics, vol. 42, no. 5, pp. 2236-2265, 2001.

[10] A. J. Davies and B. H. J. McKellar, "Nonrelativistic quaternionic quantum mechanics in one dimension," Physical Review A: Atomic, Molecular and Optical Physics, vol. 40, no. 8, pp. 42094214, 1989.

[11] A. J. Davies and B. H. J. McKellar, "Observability of quaternionic quantum mechanics," Physical Review A: Atomic, Molecular and Optical Physics, vol. 46, no. 7, pp. 3671-3675, 1992.

[12] S. De Leo, G. C. Ducati, and C. C. Nishi, "Quateraionic potentials in non-relativistic quantum mechanics," Journal of Physics A: Mathematical and General, vol. 35, no. 26, pp. 5411-5426, 2002.

[13] A. Peres, "Proposed test for complex versus quaternion quantum theory," Physical Review Letters, vol. 42, no. 11, pp. 683-686, 1979.

[14] H. Kaiser, E. A. George, and S. A. Werner, "Neutron interferometric search for quaternions in quantum mechanics," Physical Review A: Atomic, Molecular and Optical Physics, vol. 29, no. 4, pp. 2276-2279, 1984.

[15] K. Shoemake, "Animating rotation with quaternion curves," ACM SIGGRAPH Computer Graphics, vol. 19, no. 3, pp. 245254, 1985.

[16] S. L. Altmann, Rotations, Quaternions, and Double Groups, Oxford, UK, Claredon, 1986. 
[17] M. Gogberashvili, "Split quaternions and particles in $(2+1)$ space," The European Physical Journal C, vol. 74, article 3200, 2014.

[18] H. Sobhani and H. Hassanabadi, "Scattering in quantum mechanics under quaternionic Dirac delta potential," Canadian Journal of Physics, vol. 94, no. 3, pp. 262-266, 2016.

[19] H. Sobhani, H. Hassanabadi, and W. S. Chung, "Observations of the Ramsauer-Townsend effect in quaternionic quantum mechanics," The European Physical Journal C, vol. 77, article 425, 2017.

[20] H. Hassanabadi, H. Sobhani, and A. Banerjee, "Relativistic scattering of fermions in quaternionic quantum mechanics," The European Physical Journal C, vol. 77, article 581, 2017.

[21] H. Sobhani and H. Hassanabadi, "Investigation of spin-zero bosons in q-deformed relativistic quantum mechanics," Indian Journal of Physics, vol. 91, no. 1205, 2017.

[22] H. Sobhani and H. Hassanabadi, "Two-dimensional linear dependencies on the coordinate time-dependent interaction in relativistic non-commutative phase space," Communications in Theoretical Physics, vol. 64, no. 3, pp. 263-268, 2015.

[23] H. Sobhani and H. Hassanabadi, "Rashba effect in presence of time-dependent interaction," Communications in Theoretical Physics, vol. 65, no. 5, pp. 543-545, 2016. 

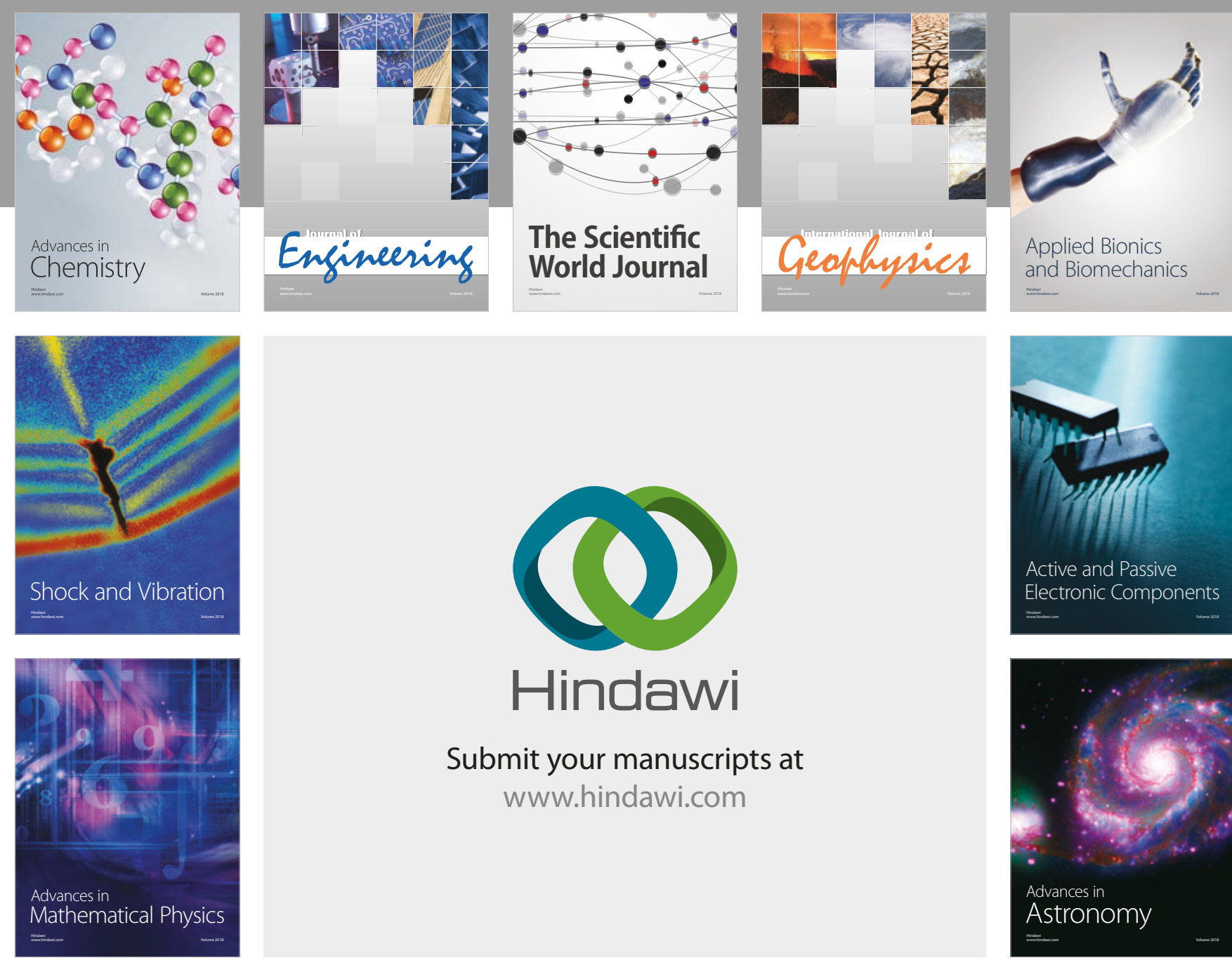

Submit your manuscripts at

www.hindawi.com

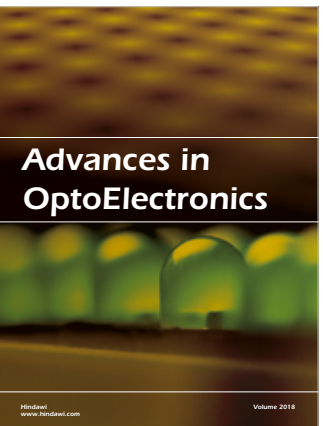

\section{Rotcting Machinery}
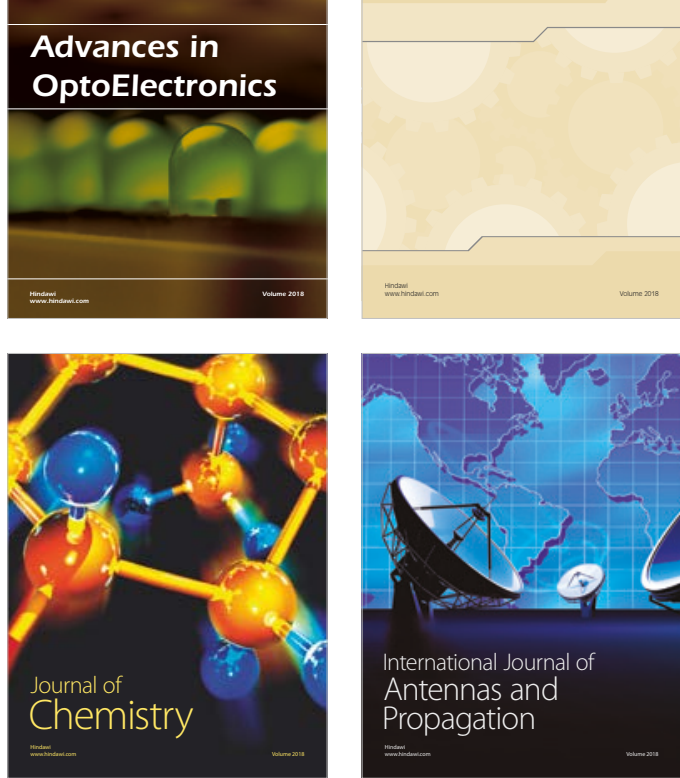

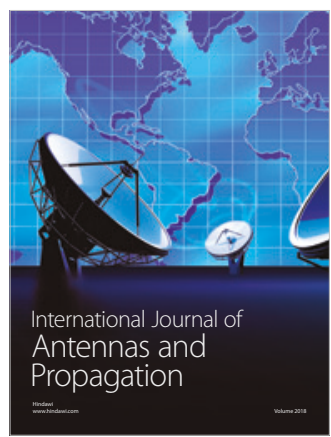

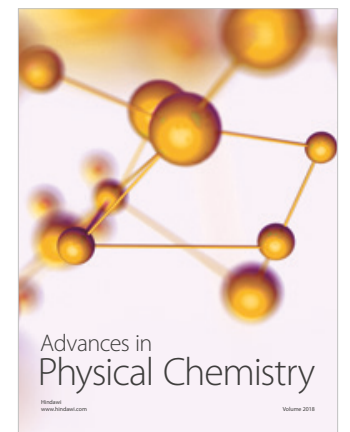

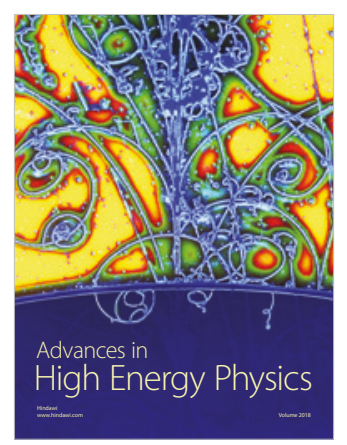

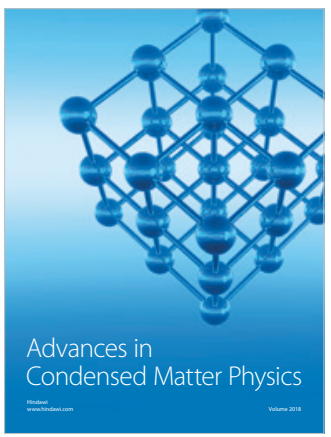

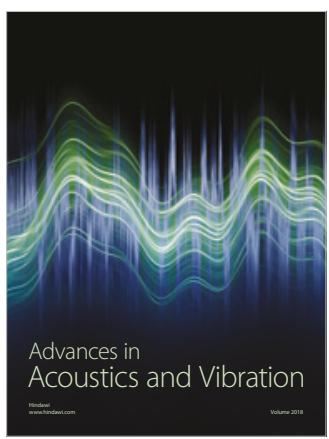

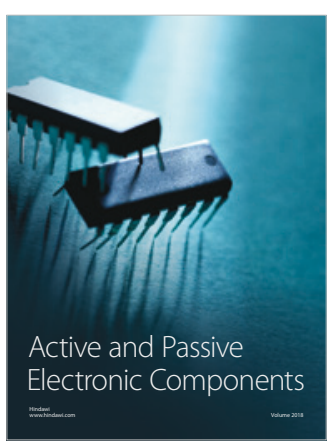
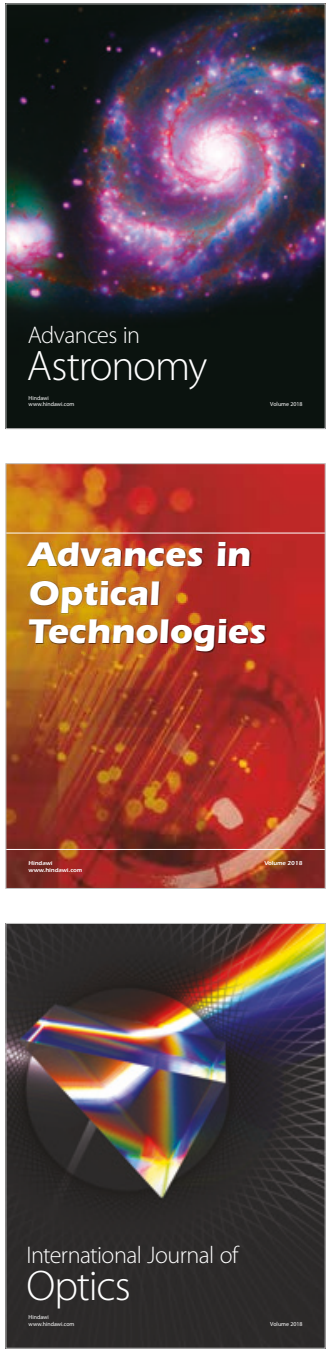